\title{
Energy Efficiency of Electrical Infrared Heating Elements
}

\author{
K.J. Brown, R. Farrelly, S.M. O’Shaughnessy, A.J. Robinson \\ Department of Mechanical \& Manufacturing Engineering, Parsons Building, Trinity College \\ Dublin, Ireland.
}

\begin{abstract}
A measurement system has been designed to characterize the radiant energy efficiency of infrared heating elements. The system also allows for measurement of the radiant heat flux distribution emitted from radiant heater assemblies. To facilitate these, a 6-axis robotic arm is fitted with a Schmidt-Boelter radiant heat flux gauge. A LabVIEW interface operates the robot and positions the sensor in the desired location and subsequently acquires the desired radiant heat flux measurement. To illustrate the functionality of the measurement system and methodology, radiant heat flux distributions and efficiency calculations are performed for a commercially available ceramic heater element for two cases. In the first, a spherical surface is traced around the entire heater assembly and the total radiant power and net radiant efficiency is computed. In the second, $50 \mathrm{~cm} \times 50 \mathrm{~cm}$ vertical planes are traced parallel to the front face of the heater assembly at distances between $10 \mathrm{~cm}$ and $50 \mathrm{~cm}$ and the in-plane power and efficiencies computed. The results indicate that the radiant efficiencies are strongly dependant on the input power to the element and, for the in-plane efficiencies, depend on the distance from the heater.
\end{abstract}

Keywords: Infrared heaters, radiation heat transfer, radiant heat flux distribution

\section{Introduction}

An infrared (IR) heater is one whose primary function is that of transferring heat to a target via the emission of infrared radiation [1]. The scope of application for radiant heaters is vast depending on the technology used. In industry, radiant heaters are used in process heating, thermoforming, curing, drying and food processing applications, to name a few [2-4]. They are also used in comfort heating for domestic applications $[5,6]$ as well as in health and medical applications ranging from incubators to postoperative rewarming [7].

Electric heating is known to be a costly form of industrial and domestic heating. However, radiant heaters offer an energy efficient and quick response form of electric heating as they operate by heating objects directly opposed to pre-heating the air surrounding the objects and transferring the heat by the less effective means of convection. An example is provided by Roth et al. [8] whereby infrared heaters used half of the energy of unit heaters in a space heating scenario in a large commercial building.

Some advantages of IR heating over conventional heating technologies include, though are not limited to the following:,

- Radiant heat exchange is between the source and the target, not the surrounding air, and can provide energy efficient heating with associated reduction in heating cost.

- Radiant heat exchange is instantaneous and requires little or no preheating. This increases cycle times in industrial applications (e.g. blow moulding of plastic drinking bottles) and makes it suitable for cost effectively heating infrequently used or large domestic and commercial spaces.

- Radiant heating systems share the same optical properties as light, i.e. the heat can be reflected, focused or diffused by reflectors, filters etc.

Radiant heaters produce different intensities and therefore different wavebands of radiation. Very high powered radiant heaters emit a high proportion of energy in the visible spectrum $(0.4-0.7 \mu \mathrm{m})$ whereby far-infrared emitting heaters $(8-14 \mu \mathrm{m})$ are generally lower powered. Further to this, the radiation heat transfer exchange between the heater and the target is also highly dependent on geometry, directionality and the surface and bulk properties of the emitter and target material. As such, the proper design of a radiant heating system or assembly requires, at the very least, an idea of the quantity of the radiant heat being emitted as well as the distribution of the heat as it spreads from the source towards the target.

Although some literature exists for gas-fired radiant heaters $[9,10]$, there exists very little available literature on the topic of characterization of electric infrared heaters, in particular those used in industrial and domestic/commercial applications. Of those which are available, the radiant heat output has been characterized by the total output power which is derived from a 2-dimensional heat flux distribution measured in a plane parallel to the heater face [11, $12]$. 
Bédard [11] has performed perhaps the only comprehensive study of IR heater characterization. In this work, three types of experiments were performed: radiant heat flux mapping/radiant efficiency evaluation, transient behaviour characterization and spectral emission characterization. For the heat flux mapping and efficiency evaluation, 13 Schmidt-Boelter heat flux gauges were arranged in a line beneath the heater under test and subsequently moved by a positioning system in order to scan and map the radiant heat flux emitted from the heater. Tests were performed for a range of gas and electrical heater types and it was shown that the radiant efficiency ranged between $39 \%$ and $85 \%$ and depended strongly on the type of heater.

More recently, Butturini \& Ngo [1] conducted a feasibility report demonstrating that the use of commercially available, though unspecified, radiant heat flux sensors to measure the heat output from a heater is a viable and repeatable method of testing the performance of electric radiant heaters. They used a manual positioning test rig and showed that the method, albeit crude, was repeatable to within $10 \%$.

As is evident from the above discussion, there exists a severe lack of information and accepted methodology for characterizing the performance of IR heating elements. Considering the penetration of IR heating technology in such a wide range of industries, spanning thermoforming to food processing, as well as the applications in domestic and commercial comfort heating, it is necessary to improve our understanding of IR heating systems, in particular their radiant energy efficiency and how the energy is spread from the source to the target.

The overarching objective of this work is to describe a measurement system and experimental methodology for measuring the net radiant output and subsequent radiant energy efficiency of IR heater assemblies. The sub-objectives are as follows:

- To determine the net radiant efficiency of a commercially available ceramic IR heating assembly over a range of input powers

- To measure the in-plane radiant efficiency of the same heater for varying distances from the heater.

- To map the heat flux distribution in 3-dimensional space in front of the heater.

- To compare the performance of radiant heater assemblies of similar geometry and rated power.

It is hoped that this work will provide a new starting point for the correct and rigorous determination of the radiant energy efficiency and heat flux distribution of electric IR heaters that can be used by heater manufacturers and end users which will assist in the design of future efficient radiant heater assemblies and systems.

\section{Experimental Apparatus, Methodology and Data Analysis}

\subsection{Experimental Apparatus}

Fig. 1 illustrates a schematic of the experimental apparatus. As it is shown, a 6-axis ABB IRB-120 robotic arm, with a position accuracy of $\pm 10 \mu \mathrm{m}$, is fixed to a Thorlabs PTM51018-AL optical table with active supports and vibration isolation. The robotic arm is connected to an ABB IRC-5 controller, through which the robotic arm's motion and position is governed. The robot controller is connected to a dedicated computer via Ethernet.

A specialized jig is fixed to the wrist of the robotic arm which holds a MedTherm 64-Series SchmidtBoelter radiant heat flux sensor. The heat flux sensor is factory calibrated over the range $0-2 \mathrm{~W} / \mathrm{cm}^{2}$ with an uncertainty of $\pm 3 \%$ of reading and is provided with a certified comparison calibration per written procedures to ANSI/NCSL Z540-1, ISO 10012-1 and ISO/IEC 17025. The calibration is traceable through temperature standards and electrical standards to the National Institute of Standards and Technology (NIST). The sensor operates by measuring a temperature difference across a material of known thermal conductivity using thermopiles on the front and back face. To eliminate convective effects, an IR transparent window is placed in front of the sensing element. The $\mathrm{ZnSe}$ window transmits electromagnetic radiation in the wavelength range of $0.5 \mu \mathrm{m}$ to $19 \mu \mathrm{m}$, which spans the emission spectrum of a of radiant heater elements [11]. Transmission losses through the window are accounted for in the factory calibration. The back side of the sensing element is water cooled by a dedicated forced water flow loop (not shown).

The heat flux sensor faces an IR heater assembly, in this case electrically powered ceramic radiant heaters. A CAD rendering of the primary element under tests is depicted in Fig. 2. The full-sized trough element (FTE) heater, manufactured by Ceramicx Ireland, is chosen here for investigation as it is a common ceramic heater used in both industrial and comfort heating applications. Other manufacturers, such as Elstein Ltd. (FSR heater series), manufacture IR heater elements of very similar design for use in the same range of applications. 
The FTE ceramic heater has a face dimension of $24.5 \mathrm{~cm} \times 6.0 \mathrm{~cm}$ with a white glaze and is heated by an imbedded coiled heater wire. An aluminium reflector is positioned at the back side of the heater to reduce losses and aid in the dispersion of the radiant heat to the frontal region. The heater is powered by an Elektro-Automatik PS8360-15 DC power supply. This power supply has a maximum output of $360 \mathrm{~V}$ or $15 \mathrm{~A}$ with voltage and current uncertainties of $\pm 0.05 \%$ and $\pm 0.15 \%$ respectively. The power supply is controlled through the main desktop computer via Ethernet. The ceramic heater under test has within it an imbedded thermocouple which is used to monitor when the heater has achieved steady state subsequent to being powered.

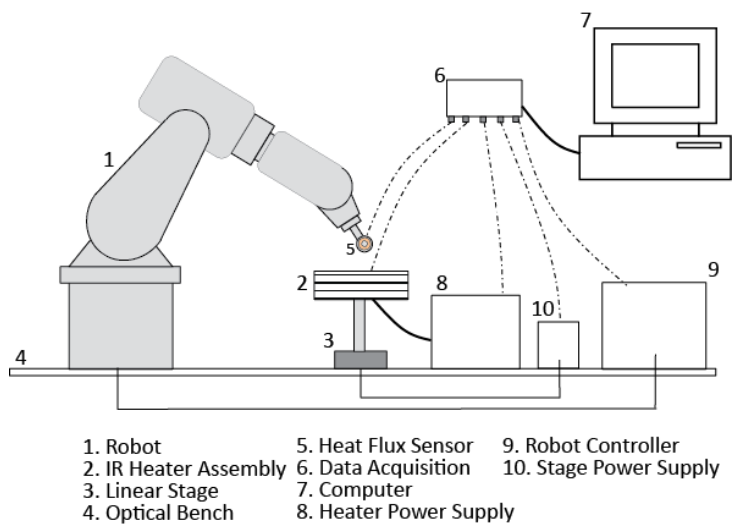

Figure 1: Schematic of the experimental facility.

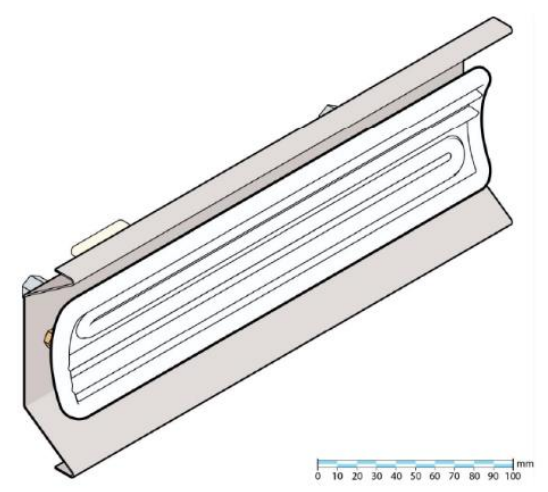

Figure 2: CAD rendering of a white glazed FTE heater element attached to aluminium reflector (Ceramicx Ltd.)

In order to have an adequate measurement range without requiring an overly large and costly robot, the heater assembly is mounted on a jig which is fixed to an Animatics XL100 linear actuator with an attached SmartMotor ${ }^{\mathrm{TM}}$ powered using a Mean Well DRP-480S power supply. The linear stage is controlled by the main computer, via an RS-232 connection, in such a way that it moves the heater element assembly towards or away from the heat flux sensor. The linear stage has a bidirectional repeatability of $\pm 16 \mu \mathrm{m}$.

\subsection{Control and Measurement Architecture}

The measurement system requires that the robot and linear stage be controlled so as to position the heat flux sensor in a specified location in front of the heater after which one or more IR heat flux measurement is acquired. The relative position and the magnitude of the sensor output voltage must then be stored for future post processing.

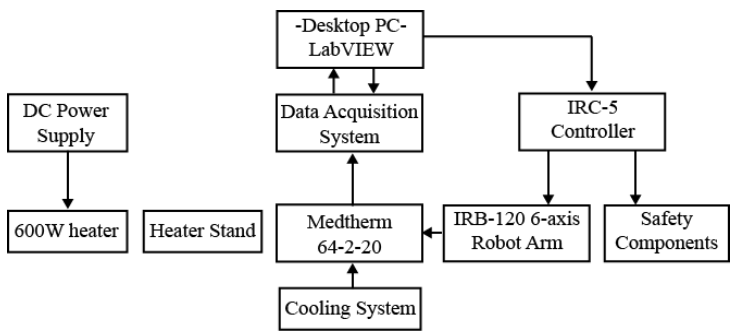

Figure 3: Schematic of the control and DAQ architecture.

Fig. 3 shows a schematic of the control and data acquisition (DAQ) architecture. For this system the control and DAQ has been centralized in LabVIEW in combination with several National Instruments analogue-to-digital (ADC) and digital to analogue (DAC) converters.

The user-operated PC is connected directly to the IRC-5 robot controller via Ethernet connection. The IRB-120 6-axis robot is governed by a series of commands implanted in the controller's database which in turn is linked to LabVIEW. The LabVIEW system administrates the operation of the robot and the data recorded from the thermocouple and heat flux sensor.

When the program within the robot controller is initiated, the robot arm defaults to a specified 'Home' position. In the LabVIEW interface, a designated grid size and resolution is defined, along with the number of measurements to be recorded at each grid point and the sampling frequency. Once these parameters have been set, the LabVIEW system sends a connection signal to the robot controller. The controller then enters a signal loop within LabVIEW, waiting to accept the next position for the robot, moving to this position and checking to ensure the position is correct. Once these steps are completed, the controller signals the LabVIEW system that the position has been reached. The DAQ system is then triggered and the desired information is recorded from the heat flux sensor. It should be noted that care must be taken to ensure sufficient time in each position to allow the sensor system to reach steady state. When the DAQ sequence has completed, LabVIEW generates the next target 
position for the robot to move to and the loop continues. At the end of a complete grid, the robot arm is signalled by the controller to move back to the 'Home' position while the linear stage is activated and traverses back to its next position. The linear stage is activated a number of times, defined by the user depending on the resolution desired.

\subsection{Data Reduction}

Two test methodologies were performed in order to gain an understanding of the radiant performance of the heater elements. The techniques and methodologies discussed here are generic, in the sense that they are applicable to any heater size and geometry. However, the present embodiment is limited by the reach of the robot which was sized specifically for smaller heater elements used extensively in industrial applications such as thermoforming. Of course larger radii, and thus larger heater systems, could be facilitated by a larger robot.

Spherical Radiant Tests: The purpose of this test is to determine the Net Frontal Radiant Efficiency (NFRE) and Net Radiant Efficiency (NRE) of the radiant heater. To facilitate these, a hemisphere of $15 \mathrm{~cm}$ radius, with origin at the geometric centre of the heater, is traced around the heater. The objective of course is to measure all of the radiant heat being emitted from the heater to a region beyond a plane which coincides with the heater face. The robot ensures that the sensor face is tangent to the surface of the sphere at all times and measurements are taken on a grid defined by $5^{\circ}$ increments. The radiant power through the hemisphere is determined by assuming that the heat flux is uniform for each grid surface element so that the power through it can be calculated by the product of the local heat flux, $\mathrm{q}^{\prime \prime}{ }_{\mathrm{ij}}$, and the elemental surface area, $\mathrm{A}^{\prime \prime}{ }_{\mathrm{ij}}$. The net power dissipated from the heater through the hemispherical surface was numerically integrated by simple addition of the elemental powers according to equation 1 .

$$
P_{H S}=\sum_{i=1}^{N} \sum_{j=1}^{N} q_{i j}^{\prime \prime} A_{i j}^{\prime \prime}
$$

The elemental surface area, $\mathrm{A}_{\mathrm{ij}}$, was a calculated as a spherical surface element with arc angles of $5^{\circ}$ such that $\sum_{i=1}^{N} \sum_{j=1}^{N} A_{i j}^{\prime \prime}=4 \pi \mathrm{R}^{2}$, where $\mathrm{R}=15 \mathrm{~cm}$.

The electrical power to the radiant heater is the product of the voltage and current to the heater,

$$
P_{e}=I V
$$

In this way the total useful radiant power emanating from the front face of the heater assembly is determined by performing measurements for a

hemisphere positioned over the front face of the heater, and the radiant losses to the rear region are likewise measured for a hemisphere on the opposite side of the heater, the two together forming a completed sphere. Thus, the NFRE is determined by,

$$
N F R E=\frac{P_{H S, \text { front }}}{P_{e}} \times 100 \%
$$

The NRE is determined in a similar way except that the measurements for the complete sphere are considered such that,

$$
N R E=\frac{P_{H S, \text { front }}+P_{H S, \text { rear }}}{P_{e}} \times 100 \%
$$

Of course, if NRE=NFRE it would indicate that there are no radiant heat losses to the rear of the heater system. Thus any discrepancy between NFRE and NRE will be indicative of the effectiveness of the reflector system.

Grid refinement tests were performed with increasing grid resolution until the PHS converged to better than $3 \%$. It was determined that $5^{\circ}$ could be considered a sufficiently refined grid.

In-Plane Radiant Tests: The aim of these tests is to visualize the heat flux distribution in front of the heater as well as determine the Net In-Plane Radiant Efficiency (NIPRE) of the heater at different distances from the heater face. The in-plane radiant power, defined here as an example, is related to the net radiant power passing through a $50 \mathrm{~cm}$ x $50 \mathrm{~cm}$ plane centred on the geometric centre of the heater element. In this way it would be related to the energy impinging on vertical target of the same dimension. For these tests each interrogation area is subdivided into a grid with a resolution of $1.0 \mathrm{~cm} \times 1.0 \mathrm{~cm}$, which was determined experimentally to be sufficiently refined in a similar way as for the Spherical Radiant Tests. Measurements are taken for planes positioned at horizontal distances of $10 \mathrm{~cm}$ to $50 \mathrm{~cm}$ from the front plane of the heater with $10 \mathrm{~cm}$ increments. The interrogation planes are illustrated in Fig. 4. For a given horizontal position the power passing through the plane and the NIPRE are calculated, respectively, as follows,

$$
P_{I P}=\sum_{i=1}^{M} \sum_{j=1}^{M} q_{i j}^{\prime \prime} A_{i j}^{\prime \prime}
$$

$$
N I P R E=\frac{P_{I P}}{P_{e}} \times 100 \%
$$




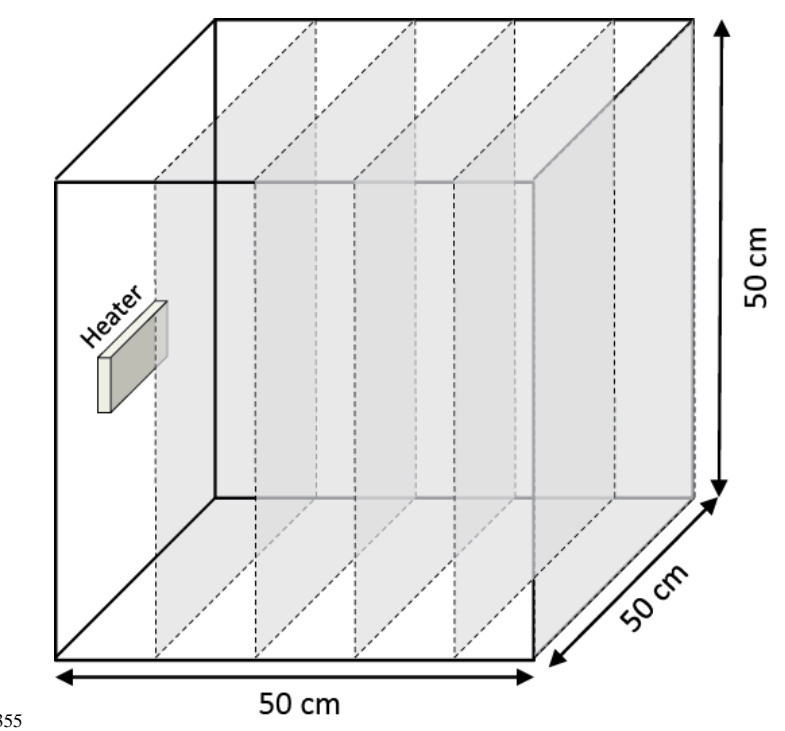

356

Figure 4: Interrogation planes for in-plane radiant tests.

\subsection{Experimental Uncertainty}

The uncertainties in the spatial positioning and primary measurements are listed in Table 1.

Table 1: Uncertainties on primary variables

\begin{tabular}{|l|l|}
\hline $\begin{array}{l}\text { Robotic } \\
\text { Arm }\end{array}$ & $\pm 10 \mu \mathrm{m}$ \\
\hline $\begin{array}{l}\text { Linear } \\
\text { Stage }\end{array}$ & $\pm 16 \mu \mathrm{m}$ \\
\hline $\begin{array}{l}\text { Heat Flux } \\
\text { Sensor }\end{array}$ & $\begin{array}{l} \pm 3 \% \text { rdg (expanded uncertainty } \\
\text { with coverage factor k=2) }\end{array}$ \\
\hline Voltage & $\pm 0.05 \%$ rdg \\
\hline Current & $\pm 0.15 \%$ rdg \\
\hline
\end{tabular}

The estimated uncertainties associated with the efficiencies depend on the power level applied to the heater and, for the NIPRE, the distance from the heater. For cases where there is a high overall power through the interrogation surface, such as the NFRE, NRE at full power and the NIPRE at full power and at the closest plane to the heater the uncertainty did not exceed $5 \%$. For the cases when the overall power throughput is low, such as the NFRE, NRE at the lowest power and the NIPRE at full power and at the furthest plane to the heater, the predicted uncertainty did not exceed $8 \%$.

\section{Results and Discussion}

\subsection{Spherical Radiant Tests}

Fig. 5 shows an example result for the heat flux distribution around the FTE heater assembly for an input electrical power of its rated $600 \mathrm{~W}$. The image shows that the heat flux is greatest in the frontal region with a visibly low proportion of the radiant heat emanating from the rear. In the front there is a fairly uniform region of heat flux, though two regions of concentrated heat flux are noticed along the equator. These are due to the closer proximity of the sensor to the heater as a result of its rectangular geometry. The net power exiting the front hemisphere was calculated to be $310.6 \mathrm{~W}$, resulting in a NFRE $=51.8 \%$. The rear region dissipates a substantially lower $29.7 \mathrm{~W}$. Combining these, this scenario results in a NRE $=56.7 \%$.

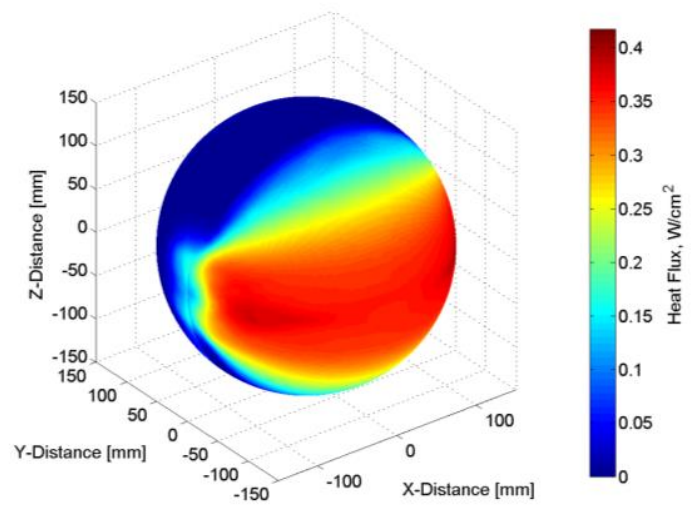

392

393 394 395 396
Figure 5: Spherical heat flux map of white FTE ceramic heater element operating with an electrical input power of $600 \mathrm{~W}$.

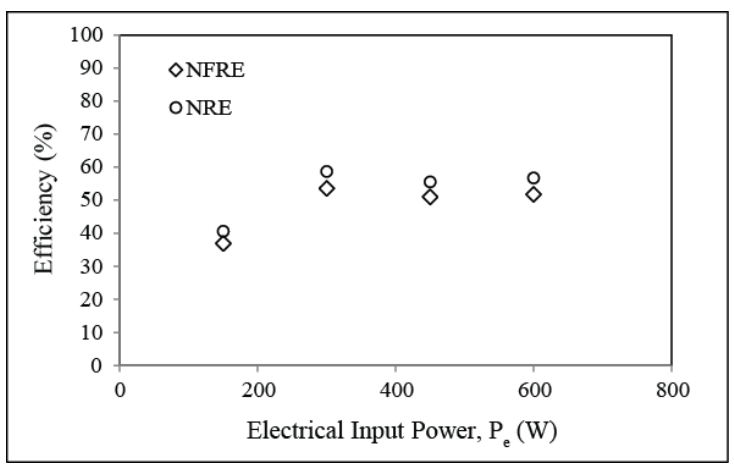

Figure 6: Net radiant and net frontal radiant efficiencies versus electrical input power for a FTE ceramic heater element.

Fig. 6 illustrates the relationship between the heater efficiencies and the electrical input power. First, there is consistently $10 \%$ more total heat radiated by the element compared with that radiated to the frontal region. Thus, the NFRE and NRE are close to one another and in the region of $50 \%$ indicating that only a portion of the heat which is radiated from the back of the element is effectively reflected and re-radiated to the frontal region by the reflector. With regard to the NRE, the measurement indicates that convective losses are approximately half of the input power. These losses would occur from both faces of the element as well as from both faces of the 


$$
R a_{L}=\left(\frac{g \beta\left(T_{s}-T_{\infty}\right) L^{3}}{\alpha v}\right)
$$

442 which can be rearranged to determine the convective

443 heat transfer coefficient, $\mathrm{h}_{\text {conv }}$.
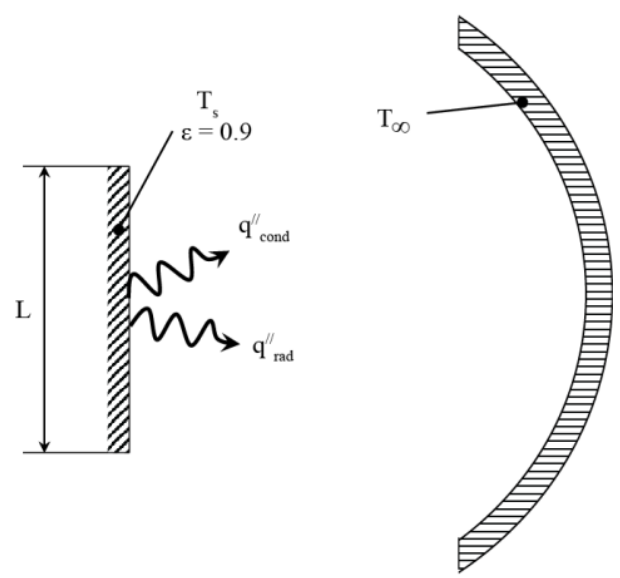

444
Figure 7: Free body diagram of the simple 1D heat transfer model.

The radiation heat transfer coefficient is defined as,

$$
h_{\text {rad }}=\varepsilon \sigma\left(T_{s}+T_{\infty}\right)\left(T_{s}^{2}+T_{\infty}^{2}\right)
$$

The heat transfer coefficients are plotted in Fig. 8 against approximated heater input power (assuming a double sided heater) and shows how the convective and radiative heat transfer coefficients are similar in magnitude at lower powers and surface temperatures. As the power and surface temperature increase the convective heat transfer coefficient increases marginally and then plateaus. The radiative heat transfer coefficient, on the other hand, continues to increase monotonically with increased power.

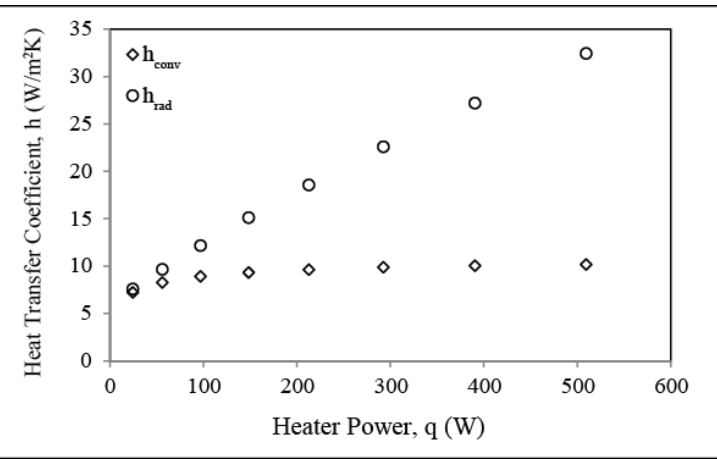

Figure 8: Approximated convective and radiative heat transfer coefficients.

The NFRE is approximated using the simplified model and is shown in Fig. 9, together with the experimentally measured efficiency. Experiments of the heater element with and without the reflector have shown that approximately $20 \%$ of the heat radiated from the rear face of the heater is reflected to the frontal region and this has been assumed in the model in determining the total radiative heat 
transferred to the front region of the heater in this model. As the figure indicates, the simple model does a surprisingly good job predicting the NFRE of the heater. In particular, the model shows the same asymptotic behaviour of the NFRE as the measured data due to the proportionally larger amount of the heat being transferred by radiation as the power level and associated surface temperature increase.

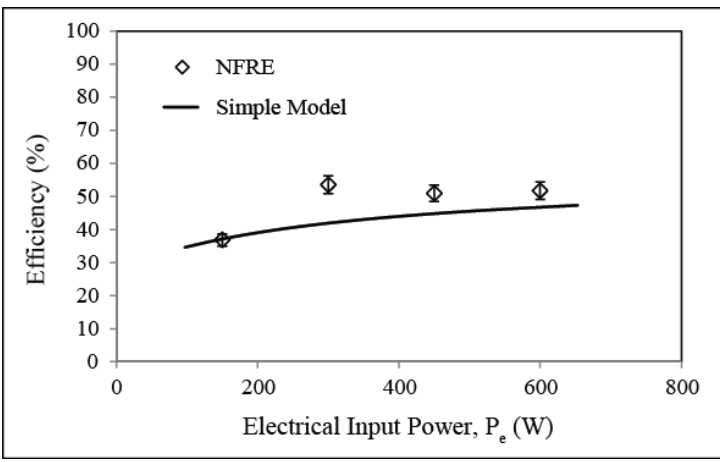

Figure 9: Predicted and measured NFRE.

\subsection{In-Plane Radiant Tests}

In application, radiant heaters, such as those under test, would be used singly or in arrays. It is therefore important to understand not only the net radiation emanating from the frontal region of the heater, but also the distribution of the radiant heat. This will give an indication of the focusing of the heat as well as how it is being dispersed. Also, for a given interrogation window size and orientation, the net radiation that is being directed towards a target and the resulting source-to-target in-plane radiant efficiency can be determined. To this end, the inplane radiant tests have been performed and an example of the 3D radiant heat flux distribution is depicted in Fig. 10 for the FTE heater with $600 \mathrm{~W}$ of electrical power input.

Clearly and unsurprisingly there is a concentration of high radiant heat flux close to the heater. This is depicted more clearly in Fig. 11a. The figure shows the $2 \mathrm{D}$ heat flux profile for a $50 \mathrm{~cm} \times 50 \mathrm{~cm}$ horizontal plane located $10 \mathrm{~cm}$ from the front of the heater. As it is shown, there is a peak of $0.61 \mathrm{~W} / \mathrm{cm}^{2}$ at the center of the plane which drops off quite rapidly resulting in a steep heat flux profile. Around the edge of the plane the heat flux has dropped to between $0.0005 \mathrm{~W} / \mathrm{cm}^{2}-0.005 \mathrm{~W} / \mathrm{cm}^{2}$ indicating that most of the radiation passes through the plane at this close proximity to the heater. This is depicted in Fig. 12 where the NIPRE can be compared to the NRFE. As it is shown the two are very close, with the NIPRE being marginally lower due to the small amount of heat radiated outside of the plane through the gap between it and the heater. The information provided here would be valuable, for example, in high heat flux applications where heater arrays would be implemented. Here the relative spacing of the heater-to-target and between heaters in the array would be dictated by the desired heat flux magnitude and uniformity.

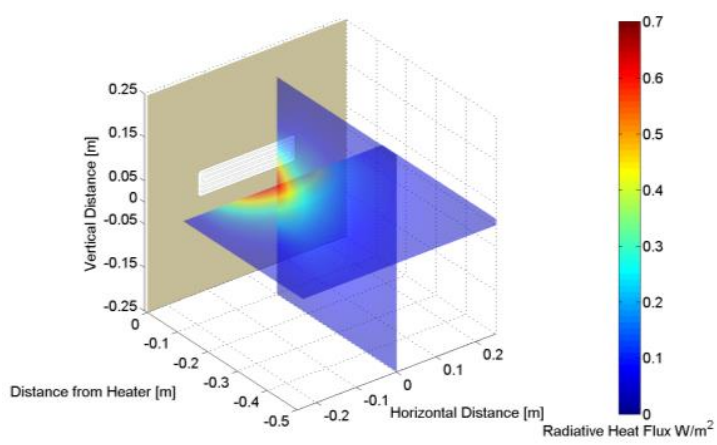

Figure 10: 3D heat flux map of FTE ceramic heater of the element operating with an electrical input power of $600 \mathrm{~W}$.

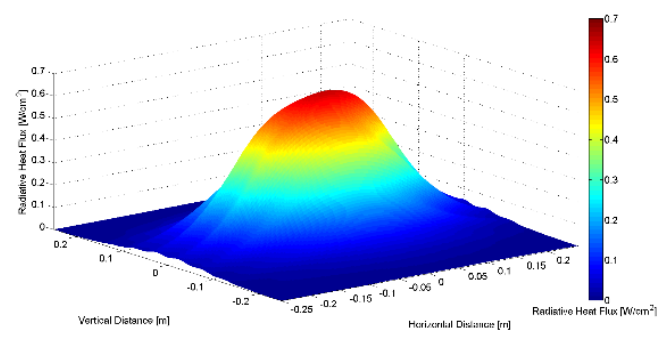

(a)

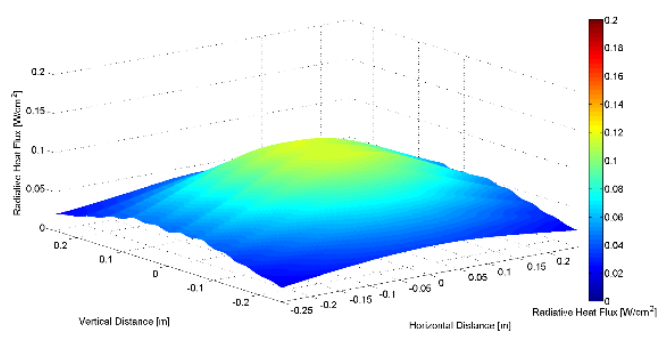

(b)

Figure 11: In-plane heat flux map of FTE ceramic heater at a location of (a) $10 \mathrm{~cm}$ and (b) $30 \mathrm{~cm}$ from the element operating with an electrical input power of $600 \mathrm{~W}$.

Fig. 10 also illustrates the diffuse nature of radiant heat transfer. Moving away from the face of the heater assembly the peak magnitude and concentration of the radiant heat flux continually decreases. This is better illustrated in Fig. 11b where the 2D heat flux distribution at a distance of $30 \mathrm{~cm}$ is depicted. Here the peak heat flux still occurs at the center of the zone, though has dropped to around $0.12 \mathrm{~W} / \mathrm{cm}^{2}$. At the edge of this particular interrogation window the levels are between 0.02 $\mathrm{W} / \mathrm{cm}^{2}$ to $0.04 \mathrm{~W} / \mathrm{cm}^{2}$ which are higher than that of 
the plane located at $10 \mathrm{~cm}$, and proportionately closer to the peak. Thus, as seen in Fig. 11b, although the heat flux levels are lower, they are more uniform, which may be advantageous in certain heating applications such as comfort heating where care must be taken to avoid skin burn injury [14] and focused heat would be undesirable.

Due to the spreading of the radiant heat to regions outside and around the viewing planes, the total heat transfer through them will decrease with linear distance from the heater. This is depicted in Fig. 12, where the decreasing net power results in a monotonically decreasing NIPRE, dropping from $47 \%$ at $10 \mathrm{~cm}$ to $15 \%$ at $50 \mathrm{~cm}$. Again, this is specific to the size of the interrogation window and would be lower for smaller areas and higher for larger ones and is given here as an illustrative example. Also shown in Fig. 12 are the results of a second test on the same heater showing the excellent repeatability.

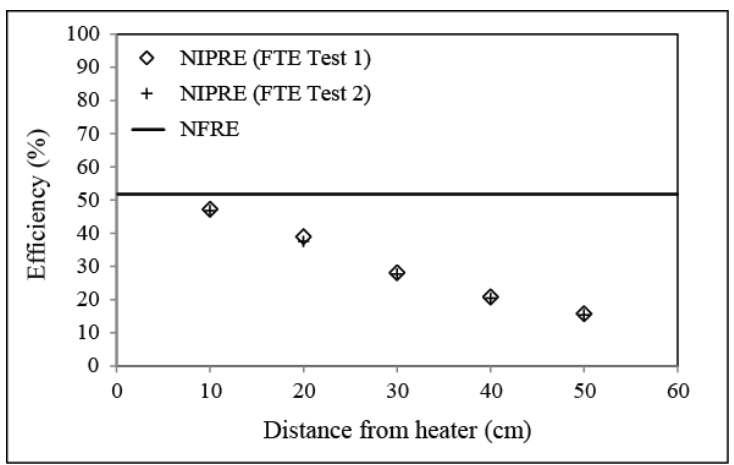

Figure 12: Net in-plane radiant efficiency at varying distances for a FTE heater with and electrical power input of $600 \mathrm{~W}$ and an interrogation area of $50 \mathrm{~cm} \mathrm{x}$ $50 \mathrm{~cm}$.

\subsection{Heater Element Design Considerations}

The above discussion describes the heat transfer and radiant energy efficiency characteristics of one particular electrical IR heater assembly. Of course the characteristics will vary between different heater designs due to aspects such as size, geometry, material composition etc. and this may vary between manufactures of ostensibly the same heating element. To illustrate the effectiveness of the measurement system and diagnostic methodology in characterizing IR heating assemblies, two additional heaters were evaluated for comparison purposes. In order to provide as close as possible to like-for-like comparisons, heaters of very similar outer dimensions and power rating were selected. To demonstrate how a heater design can influence performance, a full-sized flat hollow element (FFEH) black series heater, also manufactured by Ceramicx Ltd. was chosen since it has the same outer dimensions as the FTE heater discussed above, though in this case is flat opposed to curved, is approximately twice as thick and has a black glaze finish. Also, a FSR series ceramic heater manufactured by Elstein Ltd. is tested. This heater is ostensibly the same as the FTE heater barring that it is marginally larger, with outer dimensions of $25 \mathrm{~cm}$ $x 6.25 \mathrm{~cm}$.

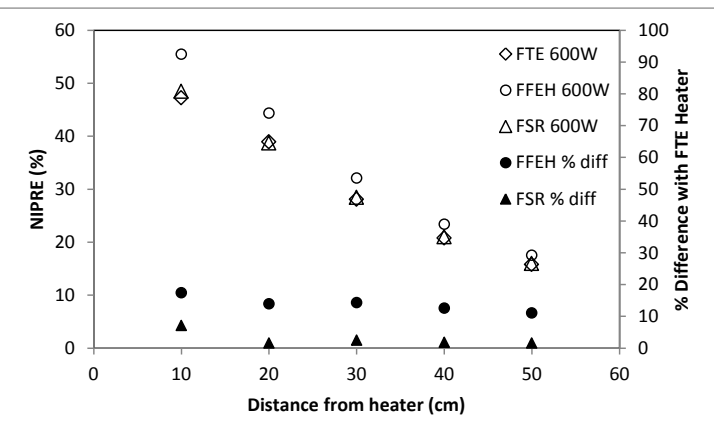

Figure 13: Net in-plane radiant efficiency at varying distances for a FTE, FFEH and FSR heater assemblies with and electrical power input of $600 \mathrm{~W}$ and an interrogation area of $50 \mathrm{~cm} \times 50 \mathrm{~cm}$.

Fig. 13 shows a comparison of the NIPRE of the two selected heater elements with the baseline heater, here the FTE series assembly. As it is shown, the FSR heater characteristic is the same as the FTE heater. The percentage difference is approximately $2 \%$ except at the closet location where the FSR is 7\% higher, which is still within experimental uncertainty. The FFEH element, however, has a notably higher NIPRE, ranging between $11 \%$ and $17 \%$.

\section{Conclusions}

This work has detailed a measurement technique and methodology for characterizing the radiant thermal performance of radiant infrared heaters and heater assemblies. As an illustrative example, a commercially available ceramic electrical heater element was tested and radiant heat flux measurements were taken for a hemispherical surfaces around the heater and for vertical planes in front of the heater. The Net Frontal Radiant Efficiency was shown to increase with initial increase in electrical power and subsequently plateau to a value in the region of around $52 \%$. The asymptotic behaviour of the NFRE is due to the insensitivity of the convective heat transfer coefficient on the heat transfer compared with the continually increasing radiative heat transfer coefficient. The Net In-Plane Radiant Efficiency at the nearest location to the heater was determined to be close to the NFRE indicating that most of the radiant heat emitted by the heater assembly to the frontal region passes through this plane. Due to the spreading of the radiant heat with distance from the source, the NIPRE continually decreases with 
distance from the heater due to continually greater amounts of radiant heat passing around the vertical planes as they get further from the source. However, although the efficiency decreases, it must be noted that uniformity of the radiant heat flux distribution improves with distance from the heater.

To illustrate how the measurement technique and methodology could be used to evaluate different radiant heater assemblies, two additional electrical heaters of ostensibly the same size and power rating were evaluated. A similar heater to the FTE, albeit from a different manufacturer, as well as a different series heater by the same manufacturer were evaluated in terms of the NIPRE. The results indicate that there is non-distinguishable difference between the same heater designs by the two different manufacturers. However, the different series heater proved to outperform the FTE element illustrating that radiant heater performance is sensitive to their geometric design, composition and glazing colour.

\section{Acknowledgements}

The authors would like to acknowledge Enterprise Ireland and Ceramicx Ltd. through grant IP-20120172. Special thanks are expressed to the European Union under and the National Development Plan 207-2013.

\section{Bibliography}

[1] Butturini R, Ngo M. Flux Mapping of Radiant Electric Heaters: Repeatability Considerations. U.S. Consumer Product Safety Commission, 2008.

[2] Leonardi SA, Viskanta R, Gore JP. Radiation and thermal performance measurements of a metal fiber burner. Journal of Quantitative Spectroscopy and Radiative Transfer.73:491-501.

[3] Schmidt FM, Le Maoult Y, Monteix S. Modelling of infrared heating of thermoplastic sheet used in thermoforming process. Journal of Materials Processing Technology. 2003,143144:225-31.

[4] Bahadori A, Vuthaluru HB. Novel predictive tools for design of radiant and convective sections of direct fired heaters. Applied Energy. 2010,87:2194-202.

[5] Laouadi A. Development of a radiant heating and cooling model for building energy simulation software. Building and Environment. 2004,39:421-31.

[6] Bojić M, Cvetković D, Bojić L. Decreasing energy use and influence to environment by radiant panel heating using different energy sources. Applied Energy. 2015,138:404-13. [7] Weyland W, Weyland A, Hellige G, Fritz U, Neumann H, Martens S, et al. Efficiency of a new radiant heater for postoperative rewarming. Acta Anaesthesiologica Scandinavica. 1994,38:601-6.

[8] Roth K, Dieckmann J, Brodrick J. Standing Columns-Emerging Technologies-Infrared Radiant Heaters. Ashrae Journal. 2007,49:72-3. [9] Mital R, Gore JP, Viskanta R. A radiation efficiency measurement procedure for gas-fired radiant burners. Experimental Heat Transfer. 1998,11:3-21.

[10] Qiu K, Hayden ACS. Increasing the efficiency of radiant burners by using polymer membranes. Applied Energy. 2009,86:349-54. [11] Bédard N. Laboratory testing of radiant gas burners and electric infrared emitters. Experimental Heat Transfer. 1998,11:255-79.

[12] Butturini R, Ngo M. Radiant electric heater flux mapping. Product Safety Engineering, 2005 IEEE Symposium on2005. p. 23-31.

[13] F.P. Incopera, D.P. De Witt. Introduction to Heat Transfer. 2nd ed: John Wiley and Sons Inc., 1990.

[14] Dai W, Wang H, Jordan PM, Mickens RE, Bejan A. A mathematical model for skin burn injury induced by radiation heating. International Journal of Heat and Mass Transfer. 2008,51:5497-510. 